\title{
Caractérisation des pesticides chimiques utilisés en production cotonnière et impact sur les indicateurs économiques dans la Commune de Banikoara au nord du Bénin
}

\author{
Prudencio T. AGBOHESSI ${ }^{1,3 *}$, Ibrahim IMOROU TOKO ${ }^{1}$, Jacob A. YABI $^{2}$, \\ Jonas F.C. DASSOUNDO-ASSOGBA ${ }^{2}$ et Patrick KESTEMONT ${ }^{3}$ \\ ${ }^{1}$ Département des Sciences et Techniques de Production Animale (STPA), Faculté d'Agronomie (FA), \\ Université de Parakou (UP), Benin. \\ ${ }^{2}$ Département d'Economie et Sociologie Rurale (ESR), Faculté d'Agronomie, Université de Parakou, Benin. \\ ${ }^{3}$ Unité de Recherche en Biologie des Organismes (URBO), Faculté des Sciences, Université de Namur, \\ Belgique. \\ *Auteur correspondant, E-mail : agbohessiprudencio@yahoo.fr
}

\section{RESUME}

Cette étude vise à caractériser les pesticides chimiques réellement utilisés en production cotonnière et à évaluer leurs impacts sur le revenu des producteurs. Pour ce faire, des données ont été collectées auprès de 90 producteurs de coton conventionnel et 40 producteurs de coton biologique choisis de façon aléatoire dans la Commune de Banikoara au nord du Bénin. Une analyse comparée entre le système de production du coton conventionnel et celui du coton biologique à l'aide de test $\mathrm{t}$ de Student de comparaison de moyennes, a été faite. Des résultats obtenus, il ressort que l'endosulfan, une matière active interdite au Bénin est encore très utilisée $(75,45 \%)$ à travers le Thionex $350 \mathrm{CE}(50,81 \%)$ et le Cotofan $350 \mathrm{CE}(24,62 \%)$ en lieu et place du Tihan 175 O-TEQ dont le taux d'adoption est encore très bas $(1,70 \%)$. L'analyse des résultats économiques révèle que les coûts totaux de production du coton conventionnel (174648 Fcfa/ha) ainsi que les rendements $(1518,2 \mathrm{~kg} / \mathrm{ha})$ sont plus élevés que ceux du coton biologique (respectivement 72930,2 Fcfa/ha et 513,9 $\mathrm{kg} / \mathrm{ha})(\mathrm{P}<0,01)$. Aussi, le produit brut, la marge nette et le revenu net du coton conventionnel à l'hectare sont meilleurs à ceux du coton biologique $(\mathrm{P}<0,05)$. La valorisation de la main d'œuvre familiale et le ratio bénéfice-coût, pour les deux types de coton amènent à conclure que la production du coton n'est pas rentable dans la localité. Toutefois, il convient de signaler que le ratio bénéfice-coût est plus élevé au niveau du coton conventionnel, lui donnant ainsi un avantage par rapport au coton biologique en termes de rentabilité hormis l'impact de l'utilisation des pesticides chimiques sur l'environnement.

(C) 2011 International Formulae Group. All rights reserved.

Mots clés : Pesticides, producteurs de coton, revenu, Banikoara, Bénin.

\section{INTRODUCTION}

La culture du coton est une activité stratégique, un des poumons de l'économie béninoise et à ce titre, elle reste incontournable dans les politiques de développement du pays. Seulement, le cotonnier est très fragile, sensible à diverses maladies et ravageurs arthropodes dont le plus redoutable est Helicoverpa armigera (Katary, 2003). De tous les outils de lutte, la méthode 
chimique est actuellement dominante avec la pulvérisation chaque année de quantités très importantes de pesticides de natures diverses. Par exemple, au cours de la seule campagne 2008-2009, pour produire 210000 tonnes de coton, plus de 1000000 litres de pesticides chimiques ont été utilisés (CSPR, 2010). La résistance des ravageurs en général et de Helicoverpa armigera en particulier aux pesticides organophosphorés et aux pyréthrinoïdes a amené les autorités béninoises à réintroduire les organochlorés à travers l'endosulfan en 1999-2000 dans le programme de traitement phytosanitaire du cotonnier (Katary, 2003). L'endosulfan, au regard de ses nuisances sur l'environnement et les populations humaines (décès de plusieurs personnes dus à l'intoxication à l'endosulfan), a été aussi remplacé par le tihan 175 O-TEQ en Février 2007 (Mbaye, 2008). Ainsi, le programme en vigueur est constitué en première fenêtre du tihan 175 O-TEQ, en deuxième fenêtre de binaires acaricides à dose faible à moyenne d'organophosphorés (dans la zone nord) et à forte dose d'organophosphorés (centre et sud) et en troisième fenêtre de produits binaires aphicides (AIC, 2009).Cependant, plusieurs études ont révélé que des différences existent entre les pesticides recommandés et ceux utilisés sur le terrain, mais il n'y a pas de données récentes sur les produits chimiques réellement utilisés (Agagbé, 2008 ; Dognon, 2008). C'est pour combler ce vide que, cette étude qui consiste à investiguer en milieu réel, auprès des producteurs de coton de Banikoara, sur les pesticides réellement utilisés dans le traitement phytosanitaire du cotonnier et l'impact de l'utilisation de ceux-ci sur les indicateurs économiques des producteurs, a été initiée. Ainsi, une étude comparée de la rentabilité du coton conventionnel et du coton biologique a été faite. La pertinence du travail réside dans le faite qu'elle s'est évertué à intégrer certains coûts supplémentaires liés à l'utilisation des pesticides chimiques dans l'analyse des résultats économiques des exploitations agricoles à base de coton, afin d'apprécier les incidences.

\section{MATERIEL ET METHODES}

\section{Zone d'étude}

La zone d'étude choisie est la Commune de Banikoara située au nord-ouest du département de l'Alibori. Elle s'étend sur une superficie de $4383 \mathrm{~km}^{2}$ dont $2148 \mathrm{~km}^{2}$ (49\%) de terres cultivables et $2235 \mathrm{~km}^{2}$ occupé par le parc W. Elle est comprise entre $10^{\circ} 50^{\prime}$ et $11^{\circ} 30^{\prime}$ latitude Nord et $2^{\circ}$ et $2^{\circ} 40^{\prime}$ longitude Est et est limitée au nord par la Commune de Karimama, au sud par les Communes de Gogounou et de Kèrou, à l'est par la Commune de Kandi et à l'ouest par la République du Burkina Faso (Figure 1). La Commune de Banikoara compte dix arrondissements (Banikoara, Founougo, Goumori, Gomparou, Kokey, Kokiborou, Ounet, Sompérékou, Soroko et Toura) et soixante neuf villages et quartiers de ville. La population totale de Banikoara est estimée à 152.028 habitants dont 75829 hommes et 76199 femmes (INSAE, 2002). La Commune est caractérisée par un climat de type soudano-sahélien avec deux saisons bien tranchées : la saison de pluie de Mai à Octobre et une saison sèche de Novembre à Avril (Gounou, 2009). La moyenne annuelle des précipitations oscille entre 723,8 à 1280,7 $\mathrm{mm}$. La végétation prédominante est composée de savanes boisées, arbustives et herbacées avec des plages d'épines aux endroits soumis à une forte influence anthropique. Les activités économiques sont basées sur l'agriculture qui occupe plus de $80 \%$, l'élevage $(15 \%)$ et le commerce $(4 \%)$ (IFDC, 2007). Cette Commune est choisie pour cette étude parce qu'elle est la plus productrice de coton au Bénin en contribuant à elle seule à près de $40 \%$ de la production nationale (Gounou, 2009). C'est donc une Commune dans laquelle il y a une très forte utilisation de pesticide chimique. 


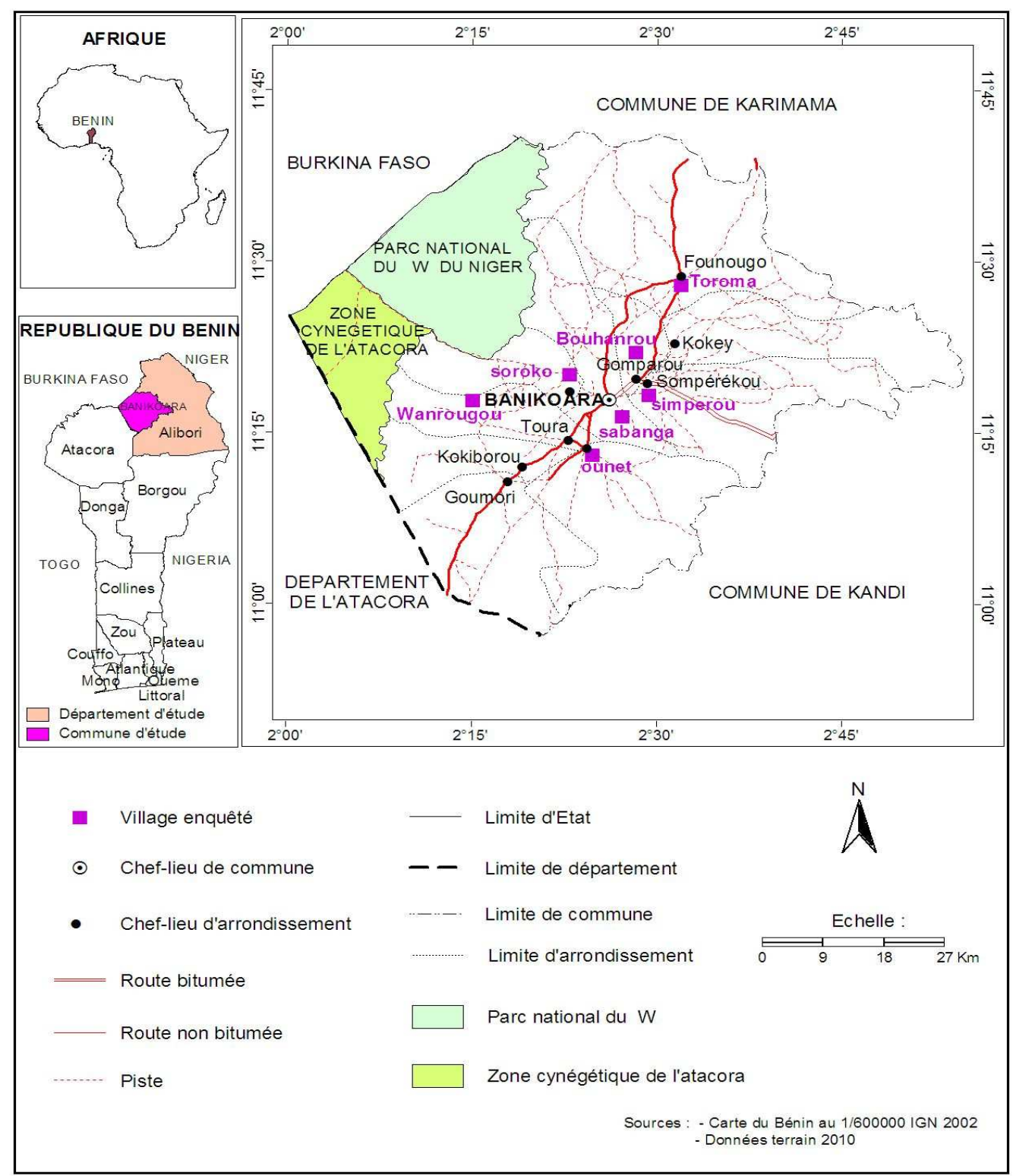

Figure 1 : Carte administrative de la Commune de Banikoara.

\section{Echantillonnage et base de données}

Les villages d'étude ont été retenus après une étude exploratoire de quinze jours faite dans la zone d'étude avec les personnes ressources telles que les membres du bureau de l'Union Communale des Producteurs de Coton (UCPC) de Banikoara, les Secrétaires Généraux des Groupements Villageois des Producteurs de Coton (GVPC), certains gros producteurs de coton, certains agents d'encadrement des producteurs, etc. Ainsi, 7 villages ont été choisis pour abriter l'enquête se basant sur trois critères à savoir : avoir produit du coton ces cinq dernières années, avoir utilisé des pesticides chimiques ces cinq dernières années, accessibilité facile des villages pendant la saison des pluies. Les villages suivants ont donc été retenus: 
Toroma, Ounet centre, Simpérou, Soroko peulh, Sabanga, Bouhanrou et Warougourou respectivement dans les arrondissements de Founougo, d'Ounet, de Somkpérékou, de Soroko, de Banikoara centre, de Gomparou et de Kokey (Figure 1).

L'unité de recherche est le producteur de coton. Un échantillonnage aléatoire a permis de choisir 130 producteurs de coton (conventionnel et biologique) à enquêter. Les villages Toroma, Ounet centre, et Simpérou ont été retenus pour enquêter les producteurs de coton conventionnel à raison de 30 producteurs par village. Quant aux producteurs de coton biologique, cinq villages ont été retenus (Simpérou, Soroko peulh, Sabanga, Bouhanrou et Warougourou) à raison de 8 producteurs par village du fait du nombre réduit de producteurs de coton biologique (350) répartis sur l'ensemble des dix arrondissements de la Commune.

L'enquête s'est déroulée pendant la période d'utilisation de pesticide dans les champs. Les investigations socioanthropologiques ont été faites par des entretiens structurés à base de questionnaire concernant essentiellement les caractéristiques de production, la production de coton, les coûts et les gains, les méthodes et coûts de traitement, etc. Aussi, des entretiens semistructurés sur la base d'un guide et des entretiens informels avec les personnes ressources au niveau communal et villageois (les membres du bureau de l'UCPC, les membres des GVPC, les techniciens du Centre Régional pour la Production Agricole (CeRPA) de Banikoara, les agents des Organisations Non Gouvernementales (ONG) intervenant dans la filière coton, etc.) et des observations directes dans certains champs, ont aidé à avoir des données relatives aux pesticides effectivement utilisés dans les champs de coton conventionnel et d'autres données relatives aux deux types de coton (conventionnel et biologique).

\section{Approches de détermination des impacts économiques des pesticides chimiques}

Les approches avant / après et sans / avec ont été développées pour mesurer les impacts de technologies introduites en production agricole.

L'approche avant/après introduction de la technologie ne permet pas d'isoler les effets liés aux facteurs exogènes à la technologie. Ainsi, cette approche peut conduire à des erreurs d'appréciation étant donné que la différence entre les situations avant et après introduction de la technologie peut être liée uniquement ou principalement aux facteurs exogènes. Quant à l'approche avec/sans, elle permet de pallier cet inconvénient et plus encore cette approche est plus conceptuellement claire (Scherr et Muller, 1991). Selon Gregersen et Contreras (1994), en appliquant le concept avec/ sans aux coûts économiques (ou coûts d'opportunité), il faut être particulièrement attentif à bien identifier la meilleure possibilité réelle abandonnée, c'est-à-dire la meilleure utilisation possible d'une ressource qui aurait effectivement été faite, en tenant compte des diverses contraintes institutionnelles. C'est pourquoi, après avoir déterminé les pesticides chimiques effectivement utilisés dans la zone d'étude, nous avons choisi l'approche méthodologique basée sur la comparaison des indicateurs économiques du système de coton conventionnel (avec utilisation de pesticides chimiques) et du système du coton biologique (sans utilisation de pesticides chimiques), puis à identifier les facteurs pouvant déterminer le revenu net du producteur.

Afin d'apprécier la performance économique de la production du coton, plusieurs indices ont été calculés. En suivant les travaux de Yabi (2010), les différents indicateurs économiques estimés par système de production sont: le Produit Brut (PB), les Coûts Fixes de production (CF), les Coûts Variables de production (CV), les Coûts Totaux de production (CT), les Marges Nettes (MN) de production, le Revenu Net (RN) de production, la valorisation nette de la main d'œuvre familiale (PML), les ratios Bénéfice/Coût de production (B/C).

\section{Le Produit Brut ou PB}

Cet indicateur représente la quantité physique obtenue à la fin de la production 
agricole. Il sera exprimé en kilogramme par hectare $(\mathrm{kg} / \mathrm{ha})$ ou en tonne par hectare (T/ha). Ainsi, le Produit Brut peut être défini par : $P B=\frac{Q}{S}$

$Q$ est la quantité produite, souvent exprimée en $\mathrm{kg}$ ou en T, et $S$ la superficie de terre utilisée exprimée en ha.

En général, pour poursuivre l'analyse de la rentabilité économique de la production, il est indispensable de disposer du prix unitaire $\mathrm{P}_{\mathrm{Q}}$ (en Fcfa par unité du produit obtenu), et d'exprimer le produit brut en valeur. Dans ce cas, le produit brut en valeur ou PBV ou encore rendement en valeur est donné par la formule: $P B V=P B * P_{Q}$

PBV est alors exprimé en Fcfa/ha

\section{Coûts Variables de production ou $\mathrm{CV}$}

Les coûts variables sont les charges de production qui sont fonction de la quantité totale produite. Ils sont liés à des décisions à court terme. En économie agricole, ces coûts sont représentés par les charges liées aux intrants agricoles (engrais, insecticides, semences, etc.), à la main-d'œuvre salariée payée et autres charges fonction de la quantité produite. Les coûts variables sont exprimés en $\mathrm{Fcfa} / \mathrm{ha}$ par :

$C V=\sum C V_{i} \quad$ Avec $C_{\mathrm{i}}$ la valeur en $\mathrm{Fcfa} / \mathrm{ha}$ du coût variable i de production.

\section{Coûts Fixes de production ou CF}

Contrairement aux coûts variables, les coûts fixes ne dépendent pas de la quantité produite. Ils sont liés à des décisions à long terme très peu réversibles. En économie rurale, ces coûts sont représentés par les éléments ci-après : intérêts payés, amortissement ou frais de location du matériel agricole, impôts et taxes payés, salaires versés et autres charges fixes. Comme décrit au niveau des coûts variables, les coûts fixes doivent être exprimés en valeur (Fcfa/ha). Ils sont mathématiquement exprimés par: $C F=\sum C F_{j}$ Avec $\mathrm{CF}_{\mathrm{j}}$ la valeur du coût fixe $\mathrm{j}$ de production en Fcfa/ha.
Dans le souci d'être aussi précis que possible dans l'estimation de l'amortissement des différents équipements utilisés dans la production du coton, nous avons procédé de la manière suivante :

$\checkmark$ Pour chaque équipement, il a été estimé le nombre moyen, la durée de vie, le prix unitaire et le coût total pour les différentes opérations culturales entrant dans la production du coton ;

$\checkmark$ L'annuité par type d'équipement a été calculée à partir de la formule suivante :

$$
\mathrm{Ami}=\frac{\mathrm{Ct}}{\mathrm{Dv}}
$$

Avec Ami : l'annuité de l'équipement considéré du producteur i, pour l'activité de production, ct : coût total de l'équipement, Dv : la durée de vie du même équipement.

\section{Coûts Totaux de production ou CT}

Les coûts totaux représentent la somme des coûts variables et fixes. Ils sont exprimés en Fcfa/ha par la formule suivante : $C T=C V+C F$

\section{La Marge Nette ou MN et le Revenu Net ou $R N$ de production}

La marge nette de production est obtenue en déduisant du produit en valeur, les coûts totaux. Elle est exprimée en Fcfa/ha par la formule suivante : $\mathrm{MN}=\mathrm{PBV}-\mathrm{CT}$

Après calcul de la marge nette, on obtient le revenu net exprimé en Fcfa par la multiplication de la marge nette par la superficie totale $\mathrm{S}$ emblavée en coton exprimée en ha. Ainsi, on a mathématiquement : $\mathrm{RN}=\mathrm{MN} * \mathrm{~S}$

\section{Valorisation nette de la main-d'ouvre familiale ou PML}

Elle est définie comme la marge nette par unité de main-d'œuvre familiale utilisée pour la production. Mathématiquement, elle est exprimée par : ${ }_{P M L}=\frac{M N}{M O}$ Avec $\mathrm{MN}$ la marge nette de l'activité de production (en $\mathrm{Fcfa} / \mathrm{ha}$ ) et MO la quantité totale de maind'œuvre familiale utilisée (homme-jour/ha). De ce fait, la valorisation nette de la main 
d'œuvre familiale est exprimée en Fcfa/homme-jour.

Dans le calcul de la quantité de maind'œuvre, trois types ont été estimés dans la production du coton dans la zone d'étude, à savoir la main d'œuvre familiale, la main d'œuvre entraide et la main d'œuvre salariale pour chaque opération de la production du coton. Les quantités de main d'œuvre ont été estimées grâce aux enquêtes quantitatives. Les fiches d'enquête utilisées à cet effet nous ont permis d'avoir des informations sur le nombre de personnes et la durée de travail. Ces deux paramètres (durée et nombre de personnes) sont déterminés par opération par type de travail et par exploitation.

Pour la détermination de la durée de travail réalisée pour chaque opération, nous avons calculé l'effectif total des travailleurs pour chaque type de main d'œuvre. L'effectif total (ET) des travailleurs en Equivalenthomme est donné par la formule suivante : $\mathrm{ET}=($ nombre d'hommes $)+0,75^{*}$ (nombre de femmes $)+0,5^{*}$ (nombre d'enfants de 6 à 14 ans).

La main d'œuvre totale (MOT) pour une opération donnée est égale à la somme de la main d'œuvre familiale MOF de la main d'œuvre entraide et de la main d'œuvre salariale (MOS). Pour la production du coton, la main d'œuvre totale est la somme de la main d'œuvre des différentes opérations effectuées durant le cycle de production.

\section{Ratio Bénéfice/Coût ou $B / C$}

$C$ 'est un indicateur d'analyse financière par excellence. Il exprime le gain financier total obtenu par l'investissement d'une unité monétaire (1 Fcfa par exemple). Si B est l'ensemble des bénéfices obtenus après un investissement total $\mathrm{C}$, on $\mathrm{a}$ : $B / C=\frac{B}{C}$

En économie agricole, $\mathrm{B}$ est désigné par le produit brut obtenu en valeur et $\mathrm{C}$ par l'ensemble de tous les coûts exprimés en valeur, y compris la main d'œuvre familiale. Ainsi, si PBV est le produit brut en valeur, CT les coûts totaux qui ne prennent pas en compte la valeur de la main-d'œuvre familiale et MOV la valeur totale de la main-d'œuvre familiale utilisée, on a :

$$
B / C=\frac{P B V}{(C T+M O V)}
$$

Les impacts de l'utilisation des pesticides sur ces indicateurs économiques sont obtenus par des tests de comparaisons de moyennes entre le groupe utilisant les pesticides et le groupe de producteurs biologiques qui n'en utilisent pas. Pour un indicateur économique $\mathrm{X}$ donné, on a théoriquement l'hypothèse nulle $\mathrm{HO}: \mathrm{m}_{1^{-}}$ $\mathrm{m}_{2}>0$ contre $\mathrm{H}_{1}: \mathrm{m}_{1}-\mathrm{m}_{2} \leq 0$, avec $\mathrm{m}_{1}$ et $\mathrm{m}_{2}$ les moyennes de l'indicateur $\mathrm{X}$ respectivement pour le groupe des producteurs utilisant les pesticides et pour le groupe ne les utilisant pas.

Pour réaliser le test, la variable $t c$ a été calculée $_{t c}=\frac{\left|\overline{\mathrm{X}_{1}}-\overline{\mathrm{X}_{2}}\right|}{S_{d}}$, qui suit une distribution $\mathrm{t}$ de Student de degré de liberté ddl $=\left(n_{1}+n_{2}\right)-2 . \quad \bar{X}_{1}$ et $\bar{X}_{2}$ sont respectivement les moyennes des échantillons du groupe des producteurs utilisant les pesticides et de celui des producteurs ne les utilisant pas. $S_{\mathrm{d}}$ est tel que :

$S_{d}^{2}=\left(\frac{n_{1}+n_{2}}{n_{1} n_{2}}\right)\left[\frac{\left(n_{1}-1\right) S_{1}^{2}+\left(n_{2}-1\right) S_{2}^{2}}{n_{1}+n_{2}-2}\right]$

Avec

$$
S_{1}^{2}=\frac{1}{n_{1}-1} \sum_{i=1}^{n_{1}}\left(\mathrm{X}_{1 \mathrm{i}}-\overline{\mathrm{X}}_{1}\right)^{2}
$$

et

$S_{2}^{2}=\frac{1}{n_{2}-1} \sum_{j=1}^{n_{2}}\left(\mathrm{X}_{2 \mathrm{j}}-\overline{\mathrm{X}}_{2}\right)^{2}$

$S_{1}^{2}$ et $S_{2}^{2}$ sont respectivement les variances des populations du groupe des producteurs utilisant les pesticides et du groupe ne les utilisant pas estimées à partir de leurs échantillons respectifs. Si tc $<\mathrm{t}(\mathrm{n} 1+\mathrm{n} 2)-2 ; 1$ $\alpha / 2$, alors on accepte l'hypothèse nulle $\mathrm{H}_{0}$, et on conclut que l'utilisation des pesticides a un 
impact positif sur l'indicateur économique considéré. Dans ce cas, l'impact est mesuré $\operatorname{par}\left(\overline{\mathrm{X}}_{1}, \overline{\mathrm{X}}_{2}\right)$

Dans la pratique, le logiciel SPSS utilisé a permis de calculer la probabilité pour qu'il existe une différence entre les moyennes. Si la probabilité $\mathrm{p}$ calculée est inférieure à 0,05 qui est le seuil de signification, en considérant le signe de la différence entre les deux moyennes, on peut alors vérifier si l'utilisation des pesticides a un impact positif ou non sur l'indicateur économique considéré.

\section{RESULTATS}

L'étude consistait à investiguer en milieu réel sur les pesticides réellement utilisés par les producteurs pour lutter contre les ravageurs du cotonnier et à dégager l'impact de l'utilisation de ces pesticides sur les indicateurs économiques de ces producteurs.

Le Tableau 1 présente les différents pesticides effectivement utilisés avec leurs matières actives et la Figure 2, la fréquence d'utilisation de chaque insecticide par rapport à la quantité totale utilisée par les producteurs de coton. De l'analyse du tableau et de la figure, il ressort que le Thionex $350 \mathrm{CE}$ dont la substance active est l'endosulfan est l'insecticide le plus utilisé à Banikoara avec $50,81 \%$ de la quantité totale d'insecticide utilisée. Il est suivi du Cotofan $350 \mathrm{CE}$ $(24,62 \%)$ toujours avec comme matière active l'endosulfan. L'endosulfan se révèle donc actuellement très fortement utilisé à Banikoara avec un taux de $75,43 \%$ de la quantité totale d'insecticide utilisée. Après viennent la Nurelle D (Cyperméthrine $(200 \mathrm{~g} / \mathrm{l})$ et Chlorpyrifos- éthyle $(36 \mathrm{~g} / \mathrm{l}))$ avec $21,03 \%$ et le Thunder 145 O-TEQ (Bètacyfluthrine (45 $\mathrm{g} / \mathrm{l})$ et Imidaclopride (100 g/l )) avec $2,47 \%$ de la quantité totale d'insecticide consommée. Quant au Tihan 175 O-TEQ (Flubendiamide $(100 \mathrm{~g} / \mathrm{l})$ et Spirotétramate $(75 \mathrm{~g} / \mathrm{l}))$, il ne représente que $1,70 \%$ de la quantité totale.

Par ailleurs, le coût de production du coton conventionnel et du coton biologique est résumé dans le Tableau 2. Si les coûts moyens engagés en intrants (fertilisants et produits phytosanitaires) et ceux de l'amortissement des matériels et équipements agricoles sur les exploitations des producteurs de coton conventionnel (respectivement 106.190 Fcfa/ha et $25.937 \mathrm{Fcfa} / \mathrm{ha}$ ) sont très significativement supérieurs à ceux engagés sur les exploitations des producteurs de coton biologique (respectivement $5.006 \mathrm{Fcfa} / \mathrm{ha}$ et12.931 Fcfa/ha) $(\mathrm{P}<0,01)$, les coûts moyens engagés sur les exploitations des producteurs de coton conventionnel pour la main d'œuvre familiale $\quad(234.340 \quad \mathrm{Fcfa} / \mathrm{ha})$ sont statistiquement plus faibles que ceux engagés sur les exploitations des producteurs de coton biologique (498.680 Fcfa/ha) ( $\mathrm{P}<0,01)$. Mais cela n'empêche pas que les coûts totaux de production du coton conventionnel à l'hectare (174.678,6 Fcfa) soient très statistiquement plus élevés que ceux engagés sur un hectare de coton biologique (72.930,20 Fcfa) $(\mathrm{P}<0,01)$.

De même, le Tableau 3 révèle que les rendements en coton-graine conventionnel $(1518,2 \mathrm{~kg} / \mathrm{ha})$ sont très significativement plus élevés que ceux en coton-graine biologique $(513,9 \mathrm{~kg} / \mathrm{ha})(\mathrm{P}<0,01)$.

Aussi, l'analyse comparée des résultats économiques du coton conventionnel et du coton biologique est récapitulée dans le Tableau 4. Si le produit brut, la marge nette et le revenu net du coton conventionnel (respectivement $289.730 \quad \mathrm{Fcfa} / \mathrm{ha}, 115.051$ Fcfa et 653.655 Fcfa) sont statistiquement plus élevés que ceux du coton biologique (respectivement 139.515 Fcfa/ha, 66.585 Fcfa et $17.983 \mathrm{Fcfa}$ ), (respectivement $\mathrm{P}<0,01$; $\mathrm{P}$ $<0,05$ et $\mathrm{P}<0,01$ ), la marge brute et la valorisation de la main d'œuvre familiale au niveau du coton conventionnel (respectivement $140.402 \mathrm{Fcfa} / \mathrm{ha}$ et 1158 Fcfa) ne sont pas significativement différentes de celle au niveau du coton biologique (respectivement $118.259,2 \mathrm{Fcfa} / \mathrm{ha}$ et 950 Fcfa) $(\mathrm{p}>0,05)$. 


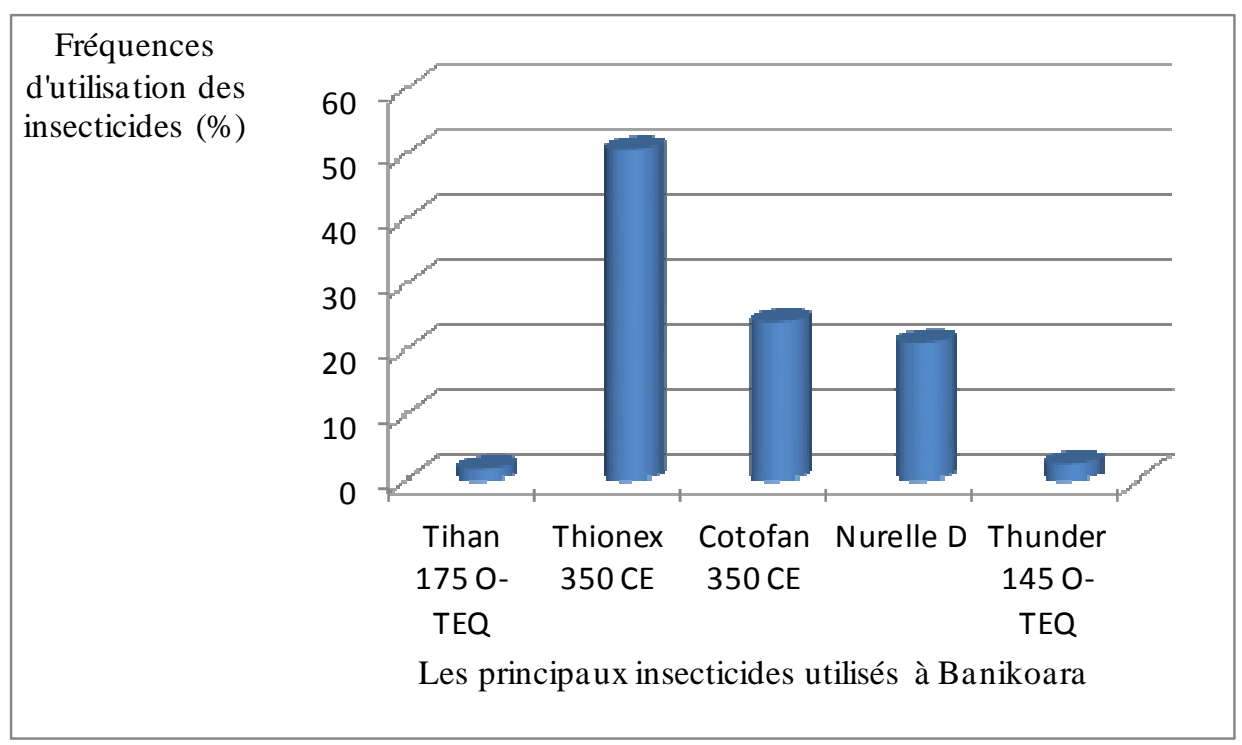

Figure 2: Pourcentage de chaque insecticide utilisé par les producteurs de coton conventionnel.

Tableau 1 : Pesticides réellement utilisés en production cotonnière.

\begin{tabular}{|c|c|c|c|}
\hline $\begin{array}{l}\text { Type de } \\
\text { pesticides }\end{array}$ & $\begin{array}{l}\text { Nom commercial ou } \\
\text { générique }\end{array}$ & Matières actives & Domaine d'utilisation \\
\hline Insecticide & Tihan 175 O-TEQ & $\begin{aligned} & \text { Flubendiamide }(100 \mathrm{~g} / \mathrm{l}) \\
+ & \text { Spirotétramate }(75 \mathrm{~g} / \mathrm{l})\end{aligned}$ & $\begin{array}{l}\text { Insecticide autorisé contre les } \\
\text { lépidoptères et les insectes } \\
\text { piqueur - suceurs du cotonnier }\end{array}$ \\
\hline Insecticide & Emir 88EC & $\begin{array}{l}\text { Cyperméthrine }(72 \mathrm{~g} / \mathrm{l})+ \\
\text { Acétamipride }(16 \mathrm{~g} / \mathrm{l})\end{array}$ & $\begin{array}{l}\text { Insecticide autorisé contre les } \\
\text { chenilles et les insectes } \\
\text { piqueur- suceurs du cotonnier }\end{array}$ \\
\hline Insecticide & Cotofan EC & Endosulfan & Insecticide non autorisé \\
\hline Insecticide & Thionex 350EC & Endosulfan & Insecticide non autorisé \\
\hline Insecticide & Nurelle D 236 CE & $\begin{array}{l}\text { Cyperméthrine }\left(\begin{array}{ll}200 & \mathrm{~g} / \mathrm{l}) \\
+ & \text { Chlorpyrifos- éthyl }(36 \\
\mathrm{g} / \mathrm{l})\end{array}\right.\end{array}$ & Insecticide autorisé \\
\hline Insecticide & Décis 25 & Deltaméthrine (25 g/l) & $\begin{array}{l}\text { Insecticide contre Helicoverpa } \\
\text { armigera }\end{array}$ \\
\hline Insecticide & Thunder 145 OD & $\begin{array}{l}\text { Bètacyfluthrine }(45 \mathrm{~g} / \mathrm{l})+ \\
\text { Imidaclopride }(100 \mathrm{~g} / \mathrm{l})\end{array}$ & $\begin{array}{l}\text { Insecticide pour les chenilles } \\
\text { carpophages, phyllophages et } \\
\text { les piqueur-suceurs du } \\
\text { cotonnier. }\end{array}$ \\
\hline
\end{tabular}




\begin{tabular}{|c|c|c|c|}
\hline Herbicide & Kalach 360SL & Glyphosate $(360 \mathrm{~g} / \mathrm{l})$ & $\begin{array}{l}\text { Herbicide systémique non } \\
\text { sélectif autorisé contre les } \\
\text { mauvaises herbes avant } \\
\text { plantation et semis de toute } \\
\text { culture. }\end{array}$ \\
\hline Herbicide & Callifor $\mathrm{G}$ & $\begin{array}{l}\text { Prométrine }(250 \mathrm{~g} / \mathrm{l}) \quad+ \\
\text { Fluaméturon }(250 \mathrm{~g} / \mathrm{l})+ \\
\text { Glyphosate }(60 \mathrm{~g} / \mathrm{l})\end{array}$ & $\begin{array}{l}\text { Herbicide systémique du } \\
\text { cotonnier utilisé en prélevé de } \\
\text { la culture et des adventices. }\end{array}$ \\
\hline
\end{tabular}

Tableau2 : Coût de production de coton utilisant ou non de pesticides chimiques (Fcfa/ha).

\begin{tabular}{|c|c|c|c|c|c|c|c|}
\hline \multicolumn{2}{|c|}{$\begin{array}{l}\text { Utilisation de } \\
\text { pesticides chimiques }\end{array}$} & $\begin{array}{l}\text { Coût total } \\
\text { engrais } \\
\text { chimiques }\end{array}$ & $\begin{array}{l}\text { Coût total } \\
\text { d'extrait } \\
\text { aqueux } \\
\text { neem }\end{array}$ & $\begin{array}{l}\text { Coût total } \\
\text { pesticide } \\
\text { chimique }\end{array}$ & $\begin{array}{l}\text { Coût total } \\
\text { main } \\
\text { d'œuvre } \\
\text { familiale }\end{array}$ & $\begin{array}{l}\text { Amortissement } \\
\text { des matériels et } \\
\text { équipements }\end{array}$ & $\begin{array}{l}\text { Coût } \\
\text { total }\end{array}$ \\
\hline \multirow{2}{*}{$\begin{array}{l}\text { Oui } \\
(\mathrm{N}=90)\end{array}$} & Moyenne & 55775 & 0 & 50415 & 234340 & 25837 & 174678 \\
\hline & Ecart-type & 29692 & 0 & 35141 & 258277 & 16429,84 & 84144 \\
\hline \multirow[t]{2}{*}{$\begin{array}{l}\text { Non } \\
(\mathrm{N}=40)\end{array}$} & Moyenne & 0 & 5006 & 0 & 498680 & 12931 & $\begin{array}{l}72930, \\
2\end{array}$ \\
\hline & Ecart-type & 0 & 2468 & 0 & 228594 & 11763,93 & $\begin{array}{l}39323, \\
76\end{array}$ \\
\hline \multicolumn{2}{|c|}{$\begin{array}{l}\text { Différence de moyenne } \\
(\mathrm{Dm})\end{array}$} & $55775^{* * *}$ & $5006 * * *$ & $50415 * * *$ & $264340 * * *$ & $12906,62 * * *$ & $\begin{array}{l}101747 \\
, 8 * * *\end{array}$ \\
\hline \multicolumn{2}{|c|}{ Test t de Student } & $\begin{array}{l}\mathrm{t}=11,85 ; \\
\mathrm{ddl}=129 ; \\
\mathrm{p}=0,00\end{array}$ & $\begin{array}{l}\mathrm{t}=-19,33 ; \\
\mathrm{dd} l=129 ; \\
\mathrm{p}=0,00\end{array}$ & $\begin{array}{l}\mathrm{t}=9,07 ; \\
\mathrm{ddl}=129 \\
\mathrm{p}=0,00\end{array}$ & $\begin{array}{l}\mathrm{t}=-5,57 ; \\
\mathrm{ddl}=129 ; \\
\mathrm{p}=0,00\end{array}$ & $\begin{array}{l}\mathrm{t}=5,08 ; \\
\mathrm{ddl}=129 ; \\
\mathrm{p}=0,00\end{array}$ & $\begin{array}{l}\mathrm{t}=7,29 ; \\
\mathrm{ddl}=12 \\
9 ; \\
\mathrm{p}=0,00\end{array}$ \\
\hline
\end{tabular}

Source : Résultat d'analyse des données d'enquête, Banikoara 2010 ; * : pas significatif ; *** : significatif au seuil de $1 \%$

Tableau 3: Moyenne du rendement en fonction l'utilisation de pesticides chimiques.

\begin{tabular}{lll}
\hline \multirow{2}{*}{$\begin{array}{l}\text { Utilisation de } \\
\text { chimiques }\end{array}$} & pesticides & \multicolumn{2}{l}{ Rendement kg/ha } \\
\cline { 2 - 3 } & Moyenne & Ecart-type \\
\hline Oui $(90)$ & 1518,2 & 888,3 \\
Non(40) & 513,9 & 187,5 \\
Différence de moyenne & $\mathrm{Dm}=1004,3 * * *$ \\
Test t de Student & $\mathrm{t}=7,06 ;$ ddl=129; $\mathrm{p}=0,00$ \\
\hline $\begin{array}{l}\text { Source }: \text { Résultats d'analyse des données d'enquête, Banikoara 2010; } \\
\text { ***: significatif au seuil de } 1 \%\end{array}$
\end{tabular}


Tableau 4 : Analyse comparée des résultats économiques du coton conventionnel et du coton biologique (Fcfa/ha).

\begin{tabular}{|c|c|c|c|c|c|c|c|}
\hline \multicolumn{2}{|c|}{$\begin{array}{l}\text { Utilisation de pesticides } \\
\text { chimiques }\end{array}$} & \multirow{2}{*}{$\begin{array}{l}\text { Produit } \\
\text { brut } \\
289730 \\
\end{array}$} & \multirow{2}{*}{$\begin{array}{l}\begin{array}{l}\text { Marge } \\
\text { brute }\end{array} \\
140402\end{array}$} & \multirow{2}{*}{$\begin{array}{l}\begin{array}{l}\text { Marge } \\
\text { nette }\end{array} \\
115051 \\
\end{array}$} & \multirow{2}{*}{$\begin{array}{l}\text { Valorisation } \\
\text { de la main } \\
\text { d'œuvre } \\
\text { familiale } \\
1158\end{array}$} & \multirow{2}{*}{$\begin{array}{l}\text { Ratio } \\
\text { bénéfice- } \\
\text { coût }\end{array}$} & \multirow{2}{*}{$\begin{array}{l}\begin{array}{l}\text { Revenu net } \\
\text { du } \\
\text { producteur }\end{array} \\
653655\end{array}$} \\
\hline Oui & Moyenne & & & & & & \\
\hline$(\mathrm{N}=90)$ & Ecart-type & 178545 & 151792 & 145618 & 1302 & 0,4 & 675220 \\
\hline Non & Moyenne & 139515 & 118289 & 66585 & 950 & 0,3 & 17983 \\
\hline$(\mathrm{N}=40)$ & Ecart-type & 50781,77 & 54433,07 & 64239,58 & 966 & 0,16 & 17901,06 \\
\hline \multirow{2}{*}{\multicolumn{2}{|c|}{$\begin{array}{l}\text { Différence de } \\
\text { moyenne }(\mathrm{Dm})\end{array}$}} & 150215,32 & 22143,26 & 48466,74 & $208,09 *$ & $0,5 * * *$ & $635672 * * *$ \\
\hline & & $* * *$ & $*$ & $*$ & & & \\
\hline \multirow{3}{*}{\multicolumn{2}{|c|}{ Test t de Student }} & $\mathrm{t}=5,21 ;$ & $\mathrm{t}=0,89$ & $\mathrm{t}=2,02 ;$ & $\mathrm{t}=0,15$ & $\mathrm{t}=-1,5$ & $\mathrm{t}=3,08 ;$ \\
\hline & & ddl $=129$; & $\mathrm{ddl}=129$; & $\mathrm{ddl}=129$; & $\mathrm{ddl}=129$; & $\mathrm{ddl}=129$ & $\mathrm{ddl}=129$ \\
\hline & & $\mathrm{p}=0,00$ & $\mathrm{p}=0,37$ & $\mathrm{p}=0,046$ & $\mathrm{p}=0,882$ & $, p=0,00$ & $\mathrm{p}=0,002$ \\
\hline
\end{tabular}

\section{DISCUSSION}

\section{Pesticides réellement utilisés en production} cotonnière

L'endosulfan est un insecticide de la famille des organochlorés cyclodiènes (PNUE, 2007) retiré du programme de traitement phytosanitaire du cotonnier au niveau de la sous région ouest-africaine en général et du Bénin en particulier depuis Février 2007, en raison de ses nuisances sur l'environnement et les populations humaines (décès de plusieurs personnes dû à l'intoxication à l'endosulfan) (Mbaye, 2008). Il est remplacé par le tihan et ne devrait donc plus être utilisé. Les produits recommandés à Banikoara depuis lors sont le tihan 175 OTEQ, Nurelle D, Thunder 145 O-TEQ et les herbicides Kalach et Callifor G (AIC, 2009). Après 3 ans d'interdiction et le délai de grâce fixé jusqu'au 31 Décembre 2008 (Mbaye, 2008), le taux d'utilisation de ce biocide reste encore très élevé $(75,43 \%)$ et le taux d'adoption du tihan très faible $(1,70 \%)$ dans la Commune la plus productrice de coton du pays. Ceci serait dû selon les producteurs, à l'inefficacité du tihan sur les ravageurs qui causerait une baisse des rendements contrairement à l'endosulfan dont les actions sont immédiatement visibles après la pulvérisation dans les champs. De plus, le tihan coûterait plus cher que l'endosulfan. En effet, un flacon de 0,1 litre de tihan coûterait 4100 Fcfa contre le même prix pour un (1) litre d'endosulfan. Cet écart entre les produits recommandés et ceux réellement utilisés, avait été aussi prouvé par Agagbé (2008) et Dognon (2008) qui ont chacun relevé dans les zones cotonnières de Savalou que seuls $36 \%$ des agriculteurs continuent de faire confiance aux produits phytosanitaires officiellement autorisés. 33\% des producteurs se ravitaillent dans le secteur informel. Le secteur informel concerne les produits issus des trafics transfrontaliers (Togo, Nigéria, Ghana, etc.) de pesticides prohibés disponibles bon marché qui échappent à tout contrôle et les produits désuets mal stockés soumis au risque de vente frauduleuse. La même observation avait aussi été faite par ANCE-Togo (2010) dans la région des savanes au Togo quand à la différence entre les produits autorisés et ceux utilisés. 
Impact de l'utilisation des pesticides chimiques sur les indicateurs économiques Coûts de production du coton conventionnel et du coton biologique

Le niveau bas des coûts moyens engagés en intrants (fertilisants et produits phytosanitaires) sur les cultures de coton biologique (5.006 Fcfa/ha) comparativement à ceux engagés sur les champs de coton conventionnel (106.190 $\mathrm{Fcfa} / \mathrm{ha})$ peut s'expliquer par le fait qu'au niveau du coton biologique, les fertilisants sont de la fumure organique comme la bouse de vache et les produits de traitement phytosanitaire des extraits de plante comme le neem (Azadirachta indica) qui ne s'achètent pas à Banikoara. A l'opposé, pour le coton conventionnel les pesticides chimiques et les engrais minéraux sont acquis à des coûts élevés. Cette observation avait été faite par l'OBEPAB (2002) lors d'une enquête réalisée à Djidja, à Glazoué et à Kandi et par Agba (2003) au cours d'une étude réalisée à Parakou, Bembérékê et Banikoara sur le coton biologique et celui conventionnel.

Par contre, les coûts moyens engagés sur les exploitations des producteurs de coton biologique pour la main d'œuvre familiale (498.680 Fcfa/ha) sont plus élevés que ceux engagés sur les exploitations des producteurs de coton conventionnel (234.340 Fcfa/ha). Ce résultat montre que le coton biologique demande plus de main d'œuvre que le coton conventionnel. Cette observation est comparable à celle faite dans la même étude d'OBEPAB (2002) réalisée à Djidja, à Glazoué et à Kandi et par Agba (2003) réalisée à Parakou, à Bembérékê et à Banikoara. Ceci est dû au fait que les opérations de fertilisation des sols par les bouses de vache par exemple, au niveau du coton biologique sont très contraignantes pour les producteurs ne disposant pas de cheptels. Ces producteurs doivent parcourir de longues distances pour obtenir la quantité de bouse de vache nécessaire à la fertilisation de leurs parcelles. Mais selon FiBL (2005) et Helvetas (2008), l'investissement en main d'œuvre n'est pas plus élevé dans les cultures biologiques, car si les agriculteurs biologiques consacrent plus de temps au désherbage, ils en perdent moins à lutter contre les ravageurs.

Le niveau élevé des coûts moyens de l'amortissement des matériels et équipements agricoles au niveau des exploitations des producteurs de coton conventionnel (25.937 Fcfa/ha) par rapport au coton biologique $(12.931 \mathrm{Fcfa} / \mathrm{ha})$ peut s'expliquer par le fait qu'au niveau du coton conventionnel, les producteurs emblavent de grandes superficies $(5,13 \pm 6,05$ ha) qui nécessitent donc de grands équipements et matériels contrairement aux producteurs biologiques qui n'emblavent que de petites superficies $(0,25 \pm 0,05 \mathrm{ha})$. Les capitaux élevés utilisés par les paysans conventionnels pour l'achat d'engrais et de pesticides chimiques, pour la rémunération de la main d'œuvre familiale sans oublier le coût élevé de l'amortissement des matériels et équipements pour les exploitations font élever aussi le coût total de production de coton conventionnel par rapport au coton biologique. Des résultats comparables avaient été trouvés par OBEPAB (2002) à Djidja, à Glazoué et à Kandi, par PAN-Africa (2002) au Sénégal, par Crole-Ree et al., (2004) au Mali et par FiBL (2005) et Helvetas (2008) en Inde central.

\section{Rendement en coton-graine des deux systèmes de production}

Les rendements en coton-graine conventionnel (1518,2 kg/ha) sont plus élevés que ceux du coton-graine biologique $(513,9$ $\mathrm{kg} / \mathrm{ha}$ ). Cette différence observée n'est pas seulement due au type de coton. En effet, d'autres raisons telles que l'encadrement technique rapproché dont bénéficie le coton conventionnel, le respect des itinéraires techniques et la fertilité initiale des terres peuvent influencer le rendement. Si des résultats semblables ont été obtenus par OBEPAB (2002) et Agba (2003) dans leurs études, FiBL (2005) et Helvetas (2008) ont trouvé en Inde central que les rendements de coton biologique sont 4 à 6 fois supérieurs à ceux du coton conventionnel. La question ici 
est de savoir si ces résultats se confirment sur le plan économique.

\section{Analyse comparée des autres indicateurs économiques du coton conventionnel et du coton biologique}

Le produit brut du coton conventionnel (289.730 Fcfa/ha) est plus élevé que le produit brut du coton biologique (139.515 $\mathrm{Fcfa} / \mathrm{ha})$. Les résultats similaires ont été rapportés par OBEPAB (2002) à Djidja, à Glazoué et à Kandi, par PAN-Africa (2002) au Sénégal où le produit brut dégagé sur un hectare de coton conventionnel est aussi supérieur à ceux dégagés sur un hectare de coton biologique. Par contre, la marge brute dégagée sur les cultures de coton conventionnel (140.402 Fcfa) est statistiquement comparable à celle dégagée sur les cultures de coton biologique $(118.259,2) \quad(\mathrm{P}>$ 0,05).Ces résultats sont contraires à ceux obtenus au Mali par CroleRee et al., (2004) et en Inde central par FiBL (2005) et Helvetas (2008) qui ont trouvé des marges brutes des cultures biologiques de coton supérieures de 30 à $43 \%$ à celles des cultures conventionnelles de coton.

Quand à la marge nette réalisée sur les exploitations de coton conventionnel (115.051 Fcfa), elle est plus élevée que celle réalisée sur les exploitations de coton biologique (66.585 Fcfa). Des résultats analogues avaient été trouvés par Agba (2003) dans son étude réalisée à Parakou, à Bembérékê et à Banikoara. Cependant, la valorisation nette de la main d'œuvre familiale réalisée sur les exploitations de coton conventionnel (1.158 Fcfa/homme-jour) statistiquement comparable à celle des exploitations de coton biologique (950 Fcfa/homme-jour) sont inférieures aux prix d'un homme-jour dans la zone d'étude qui est de 1.500 Fcfa. Donc le producteur aurait mieux fait de vendre sa force de travail que de produire. Le coton conventionnel et le coton biologique ne sont donc pas rentables $\mathrm{du}$ point de vue de la valorisation de la main d'œuvre familiale. De plus, le ratio bénéfice/coût est de 0,8 pour le coton conventionnel et de 0,3 pour le coton biologique. Cela signifie que 100 Fcfa investis dans une plantation de coton conventionnel rapportent 80 Fcfa alors qu'au niveau du coton biologique $100 \mathrm{Fcfa}$ investis rapportent $30 \mathrm{Fcfa}$. On peut donc conclure que la culture des deux types de coton n'est pas rentable dans la localité, mais le coton conventionnel semble avoir un avantage par rapport au coton biologique. Aussi, le revenu net dégagé du coton conventionnel (653.655 Fcfa) est plus élevé que celui dégagé du coton biologique (17.983 Fcfa). Ces résultats peuvent s'expliquer par la meilleure organisation dont jouit la filière du coton conventionnel par rapport au coton biologique. Cette bonne organisation est sanctionnée par les meilleurs rendements $\mathrm{du}$ coton conventionnel par rapport au coton biologique. Il faut noter que le système d'intrants à crédit accordé aux producteurs de coton conventionnel en est pour beaucoup dans ces résultats. En fait, il permet aux paysans non seulement de lutter efficacement contre les ravageurs et d'augmenter ainsi les niveaux de rendement mais aussi de relever le niveau de fertilité de leurs terres à travers l'utilisation d'engrais chimique contribuant ainsi à l'obtention de rendements plus ou moins constants au fil des années. Du côté du coton biologique, le manque d'organisation de l'activité en véritable filière ne lui permet pas d'atteindre avec facilité les performances réalisées au niveau du coton conventionnel. En effet, la culture de ce type de coton a été lancée au Bénin depuis 1996. Il lui reste encore du chemin à faire afin de pouvoir rivaliser avec le coton conventionnel dont la culture au Bénin a commencé depuis les années 60 . Il faut noter que les petites superficies emblavées par les producteurs de coton biologique ne sont pas de nature à arranger la situation. Ces différentes observations avaient été faites par OBEPAB (2002) à Djidja, à Glazoué et à Kandi et par Agba (2003) à Parakou, à Bembérékê et à Banikoara. Par contre, des observations contraires ont été faites en Inde central par Helvetas (2008) qui a trouvé que le coton biologique est nettement plus rentable 
que celui conventionnel et par Crole-Ree et al., (2004) au Mali où la culture des deux types de coton n'est pas rentable mais le coton biologique a un avantage par rapport à celui conventionnel.

\section{Conclusion}

Cette étude montre qu'après plus de trois ans d'interdiction de l'endosulfan au Bénin, ce biocide est encore très fortement utilisé à travers le Thionex et le Cotofan dans la Commune de Banikoara le "cotton belt" béninois. Après viennent dans l'ordre la Nurelle D (Cyperméthrine $(200 \mathrm{~g} / \mathrm{l})$ et Chlorpyrifos- éthyle (36 g/l)), le Thunder 145 O-TEQ (Bètacyfluthrine $(45 \mathrm{~g} / \mathrm{l})$ et Imidaclopride $(100 \mathrm{~g} / \mathrm{l}))$ et le Tihan $175 \mathrm{O}$ TEQ (Flubendiamide $(100 \mathrm{~g} / \mathrm{l})$ et Spirotétramate $(75 \mathrm{~g} / \mathrm{l}))$ vulgarisé depuis trois ans. L'étude nous a aussi permis de voir que le coton conventionnel et le coton biologique ne sont pas rentables quand nous y incluons tous les coûts variables. L'alternative pour les producteurs serait de s'orienter vers une gestion des ravageurs qui puisse préserver leur santé, celle de leurs proches et l'environnement. Toutefois, si les producteurs doivent continuer avec l'utilisation des pesticides chimiques, ils doivent utiliser les pesticides chimiques recommandés et respecter les périodes de traitement en considérant réellement la présence des ravageurs (Lutte Etagée Ciblée) et non faire une protection calendaire. Tout ceci passe par une poursuite du renforcement des capacités des producteurs.

\section{REMERCIEMENTS}

Nous remercions la Commission Universitaire pour le Développement (CUD) de la coopération belge qui a financé ce travail qui entre dans le cadre de nos travaux de recherche doctorale.

\section{REFERENCES}

AIC (Association Interprofessionnelle de Coton). 2009. Plan de Campagne Agricole Cotonnière 2009-2010 et
Perspectives 2010-2011. Presse de l'AIC : Cotonou.

Agagbé AA. 2008. Etude écotoxicochimique des résidus de pesticides dans le bassin versant de la rivière Agbado par la technique d'analyse ELISA en phase solide. Thèse d'ingénieur des travaux, option: Aménagement et protection de l'environnement. Université d'AbomeyCalavi (Bénin), p. 65.

Agba L. 2003. Analyse de la rentabilité économique de la production du coton dans quelques systèmes d'exploitation du Bénin. Thèse d'ingénieur des travaux statistiques. Université d'Abomey-Calavi (Bénin), p.212.

ANCE-TOGO (Association Nationale des Consommateurs et de l'Environnement du Togo). 2010. Rapport de l'Enquête sur l'Utilisation des Pesticides dans la Région des Savanes au Togo: cas de l'Endosulfan; Division de l'Environnement du Togo. Presse locale: Lomé.

CSPR (Centrale de Sécurisation des Paiements et Recouvrements). 2010. Evolution de la Production Cotonnière au cours des 20 Dernières Années au Bénin, Fiche Récapitulative (Bénin) : Presse du CSPR : Cotonou.

Crole-Ree SA, Diakité L, Touré M, Valenghi D. 2004. Coton Biologique au Mali : Prix à la Production et Scénarios de Production, Rapport. Presse Locale: Bamako.

Dognon RS. 2008. Devenir des produits phytopharmaceutiques utilisés en agriculture dans la Commune de Savalou: Inventaire et dosage de résidus de pesticides dans les eaux de la rivière Agbado par la technique ELISA en phase liquide. Thèse d'ingénieur des travaux, option: Aménagement et protection de l'environnement. Université d'AbomeyCalavi, (Bénin), p. 64.

FiBL (Institut de recherche de l'Agriculture Biologique). 2005. Impact de la Culture Biologique du Coton sur les Sources de Revenus des Petits Agriculteurs. Résultat 
du Projet Maikaal bioRe en Inde Central. Rapport de Consultation. Presse Locale : Inde Central.

Gregersen H, Contreras A. 1994. Evaluation Economique des Impacts des Projets Forestiers. Etude FAO forêt 106. Presse de la FAO : Rome.

Gounou A. 2009. Etude d'Impact Environnemental et Social de l'Installation d'une Réserve Villageoise dans la Commune de Banikoara. Rapport Définitif de la Mission 003/08/ED/CGP. Presse de l'Association ED, CENAGREF Fondation IGF : Cotonou.

Helvetas (Association Suisse pour la coopération internationale). 2008. Guide de Production du Coton Biologique et Equitable. Presse Azur Conseil : Ouagadougou.

IFDC (Centre International pour la Fertilité des sols et le Développement Agricole). 2007. Expériences d'Apprentissage de la Lutte Etagée Ciblée avec les Producteurs à la Base dans les Conditions de Banikoara. Rapport Technique d'Activités, Campagne 2006 - 2007. Presse Locale : Cotonou.

INSAE (Institut National de la Statistique et de l'Analyse Economique). 2002. Troisième Recensement Général de la Population et de l'Habitat (RGPH 3) au Bénin ( $2^{\text {nd }}$ édn). Presse Locale : Cotonou.

Katary A. 2003. Etude spatio-temporelle de la gestion de la résistance de Helicoverpa armigera (Hubner, 1808) aux Pyréthrinoïdes en culture cotonnière au Bénin. Thèse de Docteur d'Etat ès
Sciences Naturelles, Université d'Abomey-Calavi, Cotonou, p.202.

Mbaye DF. 2008. Interdiction de l'Endosulfan dans les Pays du Sahel en Afrique de l'Ouest. Rapport de Consultation, Groupe de Travail PAN/IPEN sur les Pesticides POPs. Presse Locale : Dakar

OBEPAB (Organisation Béninoise pour la Promotion de l'Agriculture Béninoise). 2002. Le Coton au Bénin, Rapport de Consultation sur le Coton Conventionnel et le Coton Biologique au Bénin. A Report for PAN UK's Pesticides Poverty and Livelihoods Project. Presse Locale: Cotonou.

PAN - Africa (Pesticide Action NetworkAfrica). 2002. Pesticide Poverty and Livelihoods Project, Lutte Contre les Ravageurs, Sécurité Alimentaire et Coton Biologique au Sénégal. Presse Locale: Dakar.

PNUE (Programme des Nations Unies pour l'Environnement). 2007. Convention de Stockholm sur les Polluants Organiques Persistants / Comité d'Etude des Polluants Organiques Persistants / Troisième Réunion : UNEP/POPS/ POPRC.3/5 Proposition Concernant l'Endosulfan. Presse Locale : Genève.

Scherr SJ, Mûller EU. 1991. Technology impact evaluation in agroforestry projects. Agrof. Syst., 13(3) : 235-257.

Yabi AJ. 2010. Analyse des déterminants de la rentabilité économique des activités des femmes rurales dans la Commune de Gogounou au Nord-Bénin. An. Scie. Agro. FSA-UAC, 14(2) : 221-239. 